\title{
Clinicopathological study and immunohistochemical evaluation of cyclin D1 in adenomatous polyps
}

\begin{tabular}{ccc}
\hline Tenya T. Abdulhameed $^{1}$ & Zheen O. J aff $^{1}$ & Nali Abdulkader Maaruf $^{2 *}$ \\
\hline Abstract
\end{tabular}

Background and objective: There are many histological types of colorectal polyps. Most of these polyps are benign epithelial polyps harboring very low risks of cancerous changes. Adenomatous polyps are the most known cancer precursors. Cyclin D1 gene amplification and or overexpression occurs in many human cancers. Cyclin D1 participates potentially in the multistep process of colorectal carcinogenesis. The present study aimed to assess the clinicopathological features of colorectal polyps and evaluate the significance of immunohistochemistry expression of cyclin D1 in adenomatouse polyp as a marker for predicting malignant transformation.

Methods: A total of 180 cases of colorectal polyps were collected from Rizgary teaching Hospital and some private laboratories in Erbil, Kurdistan Region, Iraq, from January 2013 to January 2018. All cases underwent polypectomy by colonoscopy for removal of the polyp. Only cases presented with a single polyp were included. The histopathological diagnosis was revised, and the polyps were divided into four subtypes; inflammatory, hyperplastic, juvenile, and adenomas. All adenoma specimens were stained using IHC technique with cyclin D1.

Results: Out of the 180 cases, the results showed that $70(38.9 \%)$ were adenomatous polyps, $56(31.1 \%)$ were hyperplastic polyps, $40(22.2 \%)$ were juvenile polyps, and $14(7.8 \%)$ were inflammatory polyps. Cyclin D1 nuclear staining was detected in $24(34.2 \%)$ adenomas. Statistical significant relations between cyclin D1 expression with male gender and with high grade dysplasia were found.

Conclusion: Adenoma was the most common type among colorectal polyps. Cyclin D1 was shown to be aberrantly expressed in colorectal adenomas and may play a role in the early stages of adenoma carcinoma development.

Keywords: Colorectal polyp; Cyclin D1; Adenoma.

\section{Introduction}

Polyps are regarded as overgrowth that elevates above the nearby mucosa. In the colon and rectum, polyps are usually slowly growing with only a minor risk of malignant transformation, mainly hyperplastic, juvenile, and inflammatory polyps, while adenomas are more likely to develop into cancer. ${ }^{1,2}$ Adenomas can be associated with cancer recurrences and the development into advanced malignancy depending on many factors as polyp number, size, histologic architecture, and grade of dysplasia. ${ }^{2,3}$ Colorectal cancer
(CRC) is the third most common cancer worldwide. ${ }^{4}$ Colorectal carcinogenesis is characterized by progressive accumulation of genetic abnormalities that needs changes in the balance between cell renewal and cell death in colorectal mucosa. ${ }^{5}$ Cyclins that complex with and activate several cyclin dependant kinases play an important role in cell proliferation and differentiation. There are groups of at least 11 cyclin genes in the human genome that fall into three categories: G1 phase cyclins, $S$ phase cyclins, and G2Im phase cyclins. Evidence for the

${ }^{1}$ Department of Pathology, College of Medicine, Hawler Medical University, Erbil, I raq.

2 Department of Anatomy and Histology, College of Medicine, Hawler Medical University, Erbil, I raq.

* Correspondence: nalimaaruf@gmail.com 
https:/ / doi.org/ 10.15218/ zjms.2021.007

oncogenic properties of deregulated cyclin D1 expression is provided by the analysis of several model system in which increased expression of cyclin D1 accelerates malignant cell transformation., ${ }^{6,7}$ The present study aimed to assess the clinicopathological features of colorectal polyps and evaluate the significance of immunohistochemistry expression of cyclin D1 in adenomatous polyp as a marker for predicting malignant transformation.

\section{Methods}

One hundred eighty cases formalin fixed paraffin embedded blocks of colorectal polyp cases removed by colonoscopy were collected from January 2013 to January 2018 in Erbil, Kurdistan region, Iraq. Clinicopathological parameters, including the age and gender of the patients and the site and size of the polyp, were obtained from the case reports in the lab. New sections were made from the most representative tissue block, which identified that contains suitable materials, then stained with haematoxylin and eosin (H\&E) and histopathologicaly revised. The study was approved by the Ethical Committee of the College of Medicine, Hawler Medical University, Erbil, Iraq. For adenoma cases, the histological type and the grade of dysplasia were assessed. Another thin $4 \mu \mathrm{m}$ section was done and submitted for immunohistochemistry, which was performed using the avidin-biotinperoxidase complex in which primarily monoclonal antibodies raised against Cyclin D1 was used and according to DakoCytomationEnVisionR+ Dual link system-HRP(DAB+) staining protocol for immunostaining. With each batch of stain, positive and negative control sections were incubated. Negative controls were obtained by omitting the primary antibody and by using $\mathrm{N}$-Universal negative control. Positive controls tissue specimens for Cyclin D1 were prepared using normal tonsil. Positive expression of Cyclin D1 gave nuclear staining of brown color. Nuclear staining was considered positive, indicated by brown granules. The distribution of Cyclin D1immunoreactivity in adenomas was quantitatively assessed. In cases with no positive cells, 0 score was achieved; for $1-10 \%, 1$ point was scored; for $11-50 \%$, 2 points were scored; for $>50 \%, 3$ points were scored. For further statistical analysis, Cyclin D1 expression was dichotomized into negative (score 0 and 1 ) and positive (score 2 and 3). ${ }^{9,10}$

Statistical Analysis: The statistical analysis was carried out by using the statistical package for the social sciences (SSPS, version 19) computer software. The Chi-square test and Fisher exact test were used to test for the association between the categorical variables. A $P$ value of $\leq 0.05$ was regarded as significant. A $P$ value of 0.01 was regarded as highly significant.

\section{Results}

A total of 180 cases of colorectal polyps were included in this study. One hundred and twelve $(62.2 \%)$ cases were male, and 68 cases $(37.7 \%)$ were female (Figure 1$)$. One hundred patients $(55.5 \%)$ were less than 50 years, and 80 patients (44.4\%) were equal or more than 50 years. The age of the cases ranged between 1.5-82 years, with a mean of 44.77 years. The anatomic locations of the polyps were divided as polyps removed from the colon (118 cases, 65.5\%) and the rectum (62 cases, $34.4 \%$ ). The histopathological diagnosis was revised, and polyps were divided into four subtypes; adenomas (70 (38.9\%) cases), hyperplastic polyp (56 (31.1\%) cases), juvenile polyp, (40 (22.2\%) cases), and inflammatory polyp (14 (7.8\%) cases) as shown in Figure 2. Regarding the size of adenoma, 47 cases $(67.1 \%)$ were polyps equal or less than one centimetre, and 23 cases $(32.9 \%)$ were polyps of more than one centimetre. The size of adenomas ranged between $0.3-5 \mathrm{~cm}$. The histologic architecture of adenomas was either villous ( 8 cases, $11.4 \%$ ), tubular (16 cases, $22.8 \%$ ), or tubulovillouse (46 cases $65.7 \%$ ), as shown in Figure 3. 


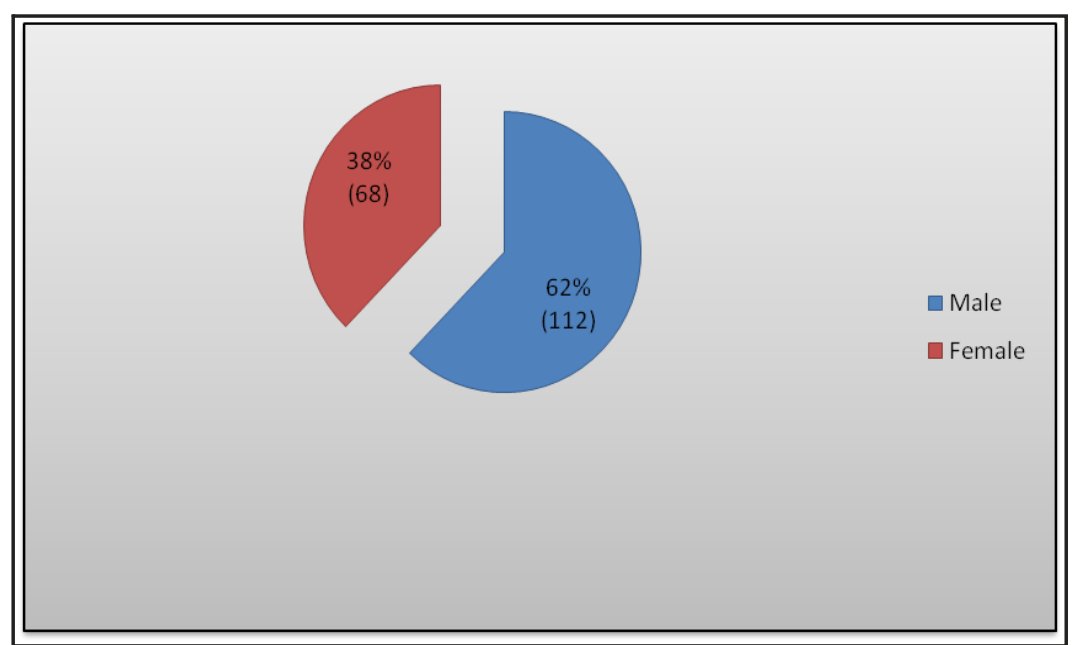

Figure 1: Distribution of Polyps according to gender.

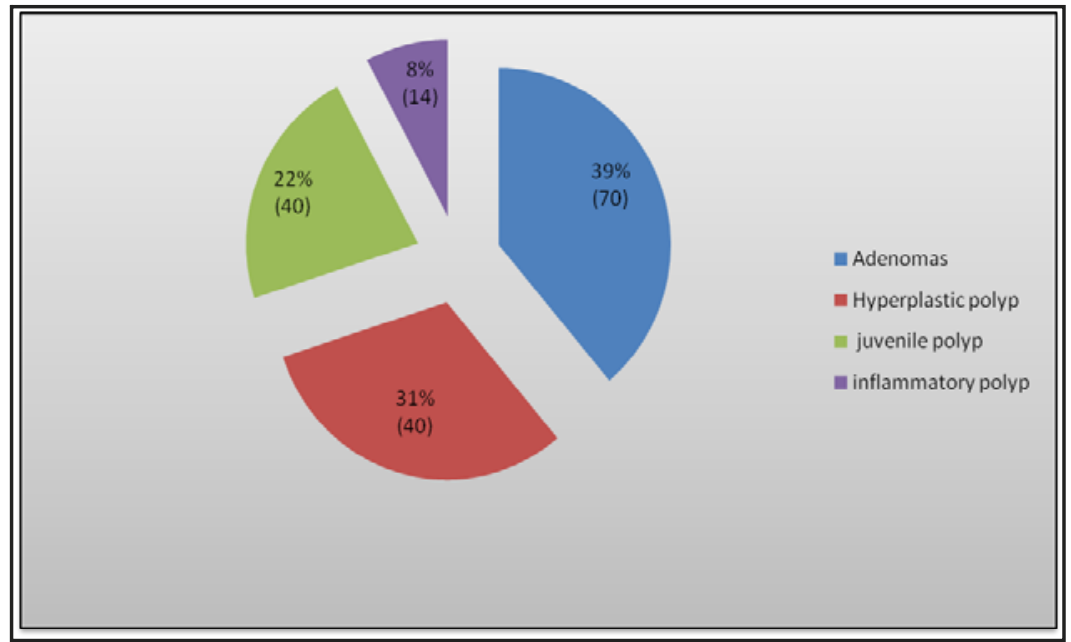

Figure 2: Histological subtypes of colorectal polyps.

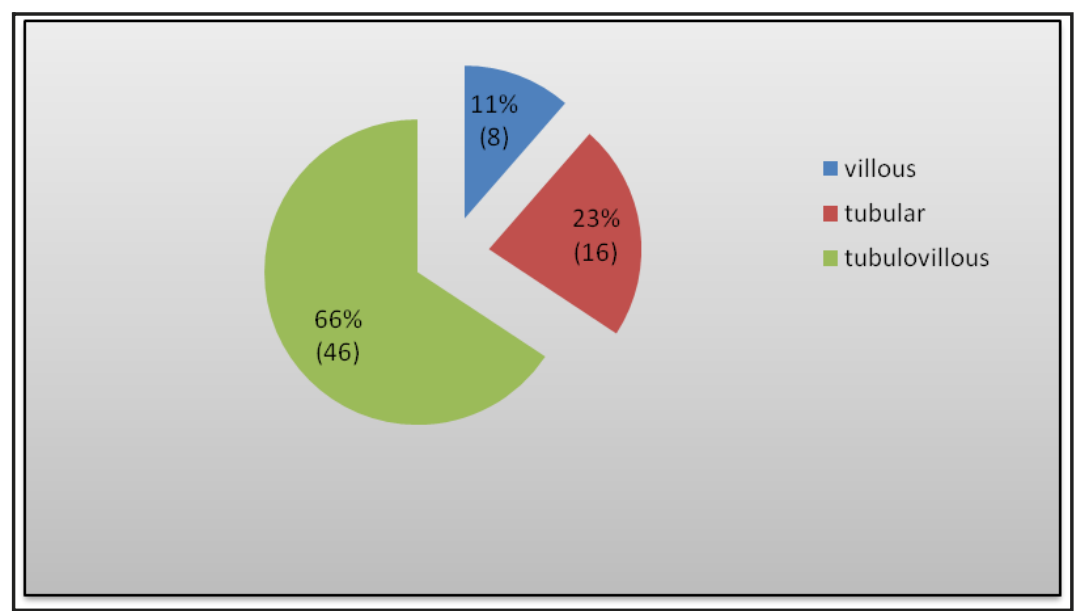

Figure 3: Distribution of colorectal adenomas according to histological architecture. 
Regarding the degree of dysplasia of adenomas, 48 cases (68.6\%) were low grade dysplasia, 22 cases $(31.4 \%)$ were high grade dysplasia. Table 1 summarizes the clinicopathological features of studied

Table1: Clinicopathologic characteristics of polyps.

\begin{tabular}{llc}
\hline Variables & Categories & No (\%) \\
\hline Age & $\geq 50$ & $80(44.4 \%)$ \\
& $<50$ & $100(55.5 \%)$ \\
Gender & Male & $112(62.2 \%)$ \\
& Female & $68(37.7 \%)$ \\
Type of polyp & Adenomatous & $70(38.8 \%)$ \\
& Hyperplastic & $56(31.1 \%)$ \\
& Juvenile retention & $40(22.2)$ \\
& Inflammatory & $14(7.8 \%)$ \\
Site of polyp & Colon & $118(65.5 \%)$ \\
& Rectum & $62(34.4 \%)$ \\
\hline
\end{tabular}

cases. Immunostaining for Cyclin D1 was performed for adenomatous polyps, 24 cases $(34.2 \%)$ were considered positive (Table 2), giving a brown nuclear staining 
pattern (Figure 4 and 5). There were no statistically significant associations between Cyclin D1 expression and the patients' age and the site, size, and type of polyp. However, a highly significant relation was achieved with the gender and the degree of dysplasia (Table 3) .

Table 3: Associations between some clinicopathologic variables and Cyclin D1 immunoreactivity in adenomatous polyps.

\begin{tabular}{|c|c|c|c|c|c|}
\hline Variables & Categories & $\begin{array}{c}\text { Total } \\
\text { No. (\%) }\end{array}$ & $\begin{array}{l}\text { Cyclin D1 } \\
\text { negative }\end{array}$ & $\begin{array}{c}\text { Cyclin D1 } \\
\text { positive }\end{array}$ & $P$ value \\
\hline \multirow[t]{2}{*}{ Age } & $\geq 50$ & $46(65.7 \%)$ & $30(65.2 \%)$ & $16(34.8 \%)$ & 0.903 \\
\hline & $<50$ & $24(34.3 \%)$ & $16(66.7 \%)$ & $8(33.3 \%)$ & \\
\hline \multirow[t]{2}{*}{ Gender } & Male & $42(60 \%)$ & $38(90.5 \%)$ & $4(9.5 \%)$ & $<0.001$ \\
\hline & Female & $28(40 \%)$ & $8(28.6 \%)$ & $20(71.4 \%)$ & \\
\hline \multirow[t]{2}{*}{ Site } & Colon & $36(51.4 \%)$ & $23(63.9 \%)$ & $13(36.1 \%)$ & 0.741 \\
\hline & Rectum & $34(48.6 \%)$ & $23(67.6 \%)$ & $11(32.4 \%)$ & \\
\hline \multirow[t]{3}{*}{ Type of adenoma } & Tubular & $16(22.8 \%)$ & $12(75 \%)$ & $4(25 \%)$ & 0.474 \\
\hline & Villous & $8(11.4 \%)$ & $4(50 \%)$ & $4(50 \%)$ & \\
\hline & Tubullovillous & $46(65.7 \%)$ & $30(65.2 \%)$ & $16(34.8 \%)$ & \\
\hline \multirow[t]{2}{*}{ Grade } & Low grade & $48(68.6 \%)$ & $39(81.3 \%)$ & $9(18.8 \%)$ & $<0.001$ \\
\hline & High grade & $22(31.4 \%)$ & $7(31.8 \%)$ & $15(68.2 \%)$ & \\
\hline \multirow[t]{2}{*}{ Size of adenoma } & $\leq 1 \mathrm{~cm}$ & $47(67.1 \%)$ & $30(63.8 \%)$ & $17(36.2 \%)$ & 0.635 \\
\hline & $>1 \mathrm{~cm}$ & $23(32.9 \%)$ & $16(69.6 \%)$ & $7(30.4 \%)$ & \\
\hline
\end{tabular}

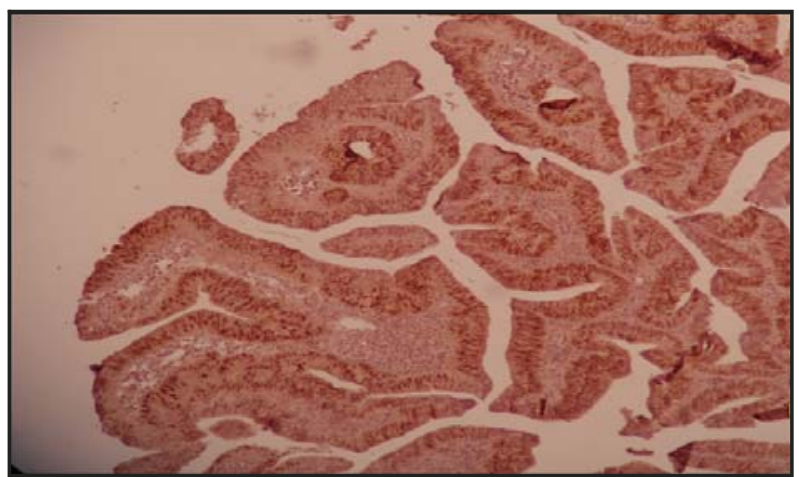

Figure 4: Positive immunohistochemical expression of Cyclin D1(score 3) in high grade adenoma.

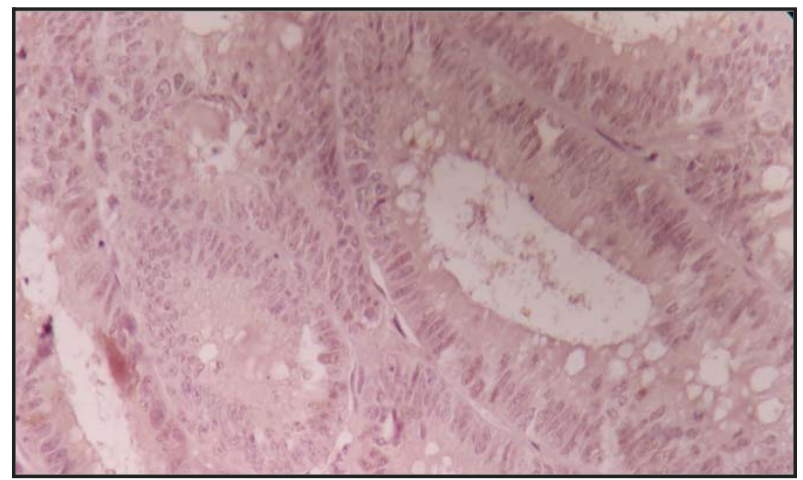

Figure 5: Negative immunohistochemical expression of Cyclin D1 (score 0) in low grade adenoma. 


\section{Discussion}

The polyp is a growth on the epithelial surface of the colon. Polypectomy currently constitutes the best strategy for preventing colorectal cancer. ${ }^{8}$ Adenomas are the most common type of colorectal polyp. Only about $15 \%$ of colorectal carcinoma develop from serrated polyp through an alternative pathway. The vast majorities develop from precursors adenoma as the most current molecular biological studies proved this concept. Many known histopathologic features are proved to be helpful as prognostic factors for adenomas. The identification of more markers is highly important to detect the development of colorectal cancer for preventive programs, including Cyclin D1 expression. ${ }^{9}$ Regarding the clinicopathological findings of the current study, it revealed that males constituted $62.2 \%$ of the studied cases, which is agreed with another study done in Iraq by Faris et al. in $2011^{10}$ and some other studies. ${ }^{11,12}$ Regarding the age, patients were ranged between 1.5-82 years with a mean of 44.77 . This finding agrees with a previous study done by Suha et al. in Iraq in $2015^{13}$ and many other studies. ${ }^{14-16}$ The age of patients with polyps is slightly younger for those with colorectal cancer, according to several previous and recent studies. ${ }^{17-19}$ This supports the concept that colonic adenomas may be the most important cause of carcinoma. If regular clinical follow up is made, adenomatous polyp could be detected and treated before the malignant transformation. This will help in avoiding the side effects of chemotherapy and decrease the morbidity and mortality associated with cancer. Many previous studies reported that distal colorectum was the prominent site of the polyp; this is nearly similar to the results of the current study. ${ }^{18,20,21}$ Regarding the type of polyp, the adenomatous polyp was the most common among the other four different types of colorectal polyps; it constitutes $38.9 \%$ of the total studied cases. A study done in Iraq found a slightly lower prevalence of adenomas in that out of 50 polyps, 16 were adenomas. ${ }^{13}$ Most adenomas were less than $5 \mathrm{~cm}$ in size; these results are in concordance with many other studies. ${ }^{10,22}$ Among these adenomas, tubulovillous (mixed architecture) is the most prevalent type constituting $65.7 \%$, followed by tubular $22.8 \%$. Similar results were obtained in Iraq, ${ }^{10}$ but another study in Iraq found that tubular pattern has the greatest frequency affecting $23(59 \%)$ cases. ${ }^{20}$ A similar result of a more tubular prevalence pattern of polyps was obtained by Sousa et al. in $2012 .^{22}$ These slight discrepancies may be due to differences in sample size and case selection bias. Regarding the degree of dysplasia, there were $48(68.6 \%)$ cases of low grade dysplasia in contrast to $22(28.5 \%)$ cases of high grade dysplasia. This result is in agreement with other studies done in Iraq. ${ }^{10,20}$ Another study from Brazil also found that $66 \%$ of adenomas were of low grade type. ${ }^{22}$ Cyclin D1 nuclear staining was detected in $24(34.2 \%)$ adenomas. This is nearly similar to the result of the study done by Farisetal. ${ }^{10}$ Another study done in Turkey showed that cyclin D1 expression is significantly increased in neoplastic polyps when compared with normal mucosa. ${ }^{9}$ However, Ayhan $^{23}$ detected Cyclin D1 expression in only $9.1 \%$ of adenomas, which may be due to different way of marker analysis. Cyclin D1 is an important regulator of cell cycle progression and can function as a transcriptional co regulator. The over expression of Cyclin D1 has been linked to the progression to cancer.6 Therefore, over expression of cyclin D1 may be a clue for dysplasia and early malignant changes in the neoplastic polyp. In the assessment of relationships between Cyclin D1 immunohistochemical expressions and studied clinicopathologic features, the current study revealed a highly significant association between gender and Cyclin D1 protein expression. The most recent studies did not find any relation between Cyclin D1 expression 
and gender, which may be due to racial and geographical factors and population differences. ${ }^{21,22}$ As regards the age, site, size, and morphological type of adenoma in the present study, there were no significant associations between them and cyclin D1 expression, and a previous study obtained similar findings. ${ }^{23}$ However, another study found a significant association between D1 expression and villous type adenoma. ${ }^{20}$ The same study also found a significant association between cyclin D1 expression and high grade dysplasia. This finding is similar to the result of this study. However, some other studies disagreed in that they found no association between Cyclin D1 expression and the degree of dysplasia. $^{20,23}$

\section{Conclusion}

Among different types of colonic polyps, adenoma is the most prevalent. Increased Cyclin D1 expression occurs in about one third of colonic adenomas with significant association with severe dysplasia, confirming its role as an early event of colonic carcinogenesis.

\section{Competing interests}

The authors declare no competing interests.

\section{References}

1. Alan S, James L, Lan S, Damjanov I. Core Pathology $3^{\text {rd }}$ ed. USA: Elsevier; 2009. P. 262-6.

2. David A, Douglas $K$, Sidney $J$, Francis $M$, David A, Theodore R. US Multi Society Task Force on Colorectal Cancer. American Cancer Society Guidelines for colonoscopy surveillance after polypectomy: a consensus update by the US Multi-Society Task Force on Colorectal Cancer and the American Cancer Society. Gastroenterology 2012; 143:844-57.

3. Kumar V, Abbas AK, Fausto N. Robbins, Cotran. Pathologic Basis of Disease $8^{\text {th }}$ ed. USA: Elsevier; 2010. P. 819-22.

4. Paulo CC, Simone SC, Luis FM. Bcl-2 Expression in rectal Cancer. Arq Gastroenterol 2006; 43 (4):284-7.

5. Bousserouel S, Lamy V, Gossé F, Lobstein A, Marescaux J, Raul F. Early modulation of gene expression used as a biomarker for chemoprevention in a preclinical model of colon carcinogenesis. Pathol Int 2011; 61:80-7.
6. Watanabe $M$, lizumi $Y$, Sukeno M, lizuka-Ohashi $M$, Sowa $Y$, Sakai $T$. The pleiotropic regulation of cyclin D1 by newly identified sesaminol-binding protein ANT2. Oncogenesis 2017; 6(4):e31

7. Zhang $Y$, Wang S, Qian W, Ji D, Wang $O$, Zhang Z, et al. uc.338 targets p21 and cyclin D1 via PI3K/AKT pathway activation to promote cell proliferation in colorectal cancer. Oncol Rep 2018; 40(2):1119-28.

8. Navarro M, Nicolas A, Ferrandez A, Lanas A. Colorectal cancer population screening programs worldwide in 2016. World J Gastroenterol 2017; 23(20):3632-42.

9. Toru S, Bilezikçi B. Early Changes in Carcinogenesis of Colorectal Adenomas. West Indian Med J 2012; 61(1):10-6.

10. Faris L, Hussam H, Haider G, Raghad J. Immunohistochemical expression of ki-67 and p53 in colorectal adenomas: A clinicopathological study. Oman Med J 2011; 26(4):229-34.

11. Khatibzadeh N, Ziaee S, Rahbar N, Molanie S, Arefian L, Fanaie $S$. The indirect role of site distribution in high-grade dysplasia in adenomatous colorectal polyps. J Cancer Res Ther 2005; 1(4):204-7.

12. Andrey B, Alexander D. Peculiarities of vascular component of communicative systems in rectal adenomas. International Journal of Collaborative Research on Internal Medicine \& Public Health 2009; 1(1):12-21.

13. Suheil SS, Mahdi LH. The Expression of P53 and ki67 in Colonic Polyps. Kufa Journal for Nursing Sciences 2015; 5(2):1-8.

14. Aune D, Chan DS, Lau R,Doris S, Rosa Lau. Dietary fibre, whole grains, and risk of colorectal cancer: systematic review and dose March 2014 Dietary Fiber and Colorectal Adenoma 697 CLINICAL AT response meta-analysis of prospective studies. BMJ 2011; 9(1):343-57.

15. Mohammad SF, Mohammad RK. Rectal cancer: a review. Med J Islam Repub Iran 2015; 29:171.

16. Ban J, Hussam H, Alaa G. Immunohistochemical expression of matrix metalloproteinase-7 in human colorectal adenomas using specified automated cellular image analysis system: a clinicopathological study. Saudi J Gastroenterol 2013; 19(1):23-7.

17. Brenner $H$, Hoffmeister M, Haug U. Should colorectal cancer screening start at same age in European countries? Contributions from descriptive epidemiology. $\mathrm{Br} \mathrm{J}$ Cancer 2008; 99(3):532-5.

18. Ulrich $\mathrm{M}$, Alexander $\mathrm{C}$, Volkmar $\mathrm{H}$, Christine $\mathrm{A}$, Volker A, Berndt B, et al. Epidemiology and quality of control of 245000 outpatient colonoscopies. Dtsch Arztebllnt 2009; 105 (24):434-40.

19. Santos JCM. Ano-rectal-colic cancer. Current Issues II - Colorectal cancer - Risk factors and prevention. Rev Bras Coloproctol 2007; 27:459-73. 
20. Faris L, Hussam HA, Ban JQ. Immunohistochemical expression of cyclin D1 in colorectal adenomas: a clinicopathological study. Kasr Al Ainy Med J 2016; 22(3):115-22.

21. Shruti $P$, Seth $L$, Huafeng $S$, Alex B, Jeff $S$, Josh $M$, et al. The association of $H$. pylori and colorectal adenoma: does it exist in the US Hispanic population. J Gastrointest Oncol 2014; 5(6):463-8

22. Sousa W, Rodrigues L, Silva R, Vieira F. Immunohistochemical evaluation of P53 and $\mathrm{Ki}-67$ proteins in colorectal adenomas. Arq Gastroenterol 2012; 49(1):35-9.

23. Ayhan $S$, Isisag $A$, Saruc $M$, Nese $N$, Demir MA, Kucukmetin NT. The role of $p R B$, p16 and cyclin D1 in colonic carcinogenesis. Hepatogastroenterology 2010;57(98):251-6. 